\title{
Vivências de pessoas submetidas à cirurgia bariátrica: base empírica para o cuidar em enfermagem
}

\author{
Experiences of people undergoing bariatric surgery: an empirical basis for nursing care \\ Experiencias de personas sometidas a cirugía bariátrica: una base empírica para el \\ cuidado de enfermería
}

Rafaella Martins Freitas Rocha ${ }^{1}$, Igho Leonardo do Nascimento Carvalho', Erisonval Saraiva da Silva ${ }^{1}$, Ana Lívia Castelo Branco de Oliveira ${ }^{1}$, Nuno Damácio de Carvalho Félix ${ }^{2}$, Ingrid Moura de Abreu $^{1 *}$, Amanda Vieira Sarmento ${ }^{1}$, Ellen Karem Rodrigues da Silva ${ }^{1}$, Aberlam da Silva ${ }^{1}$, Pâmela Pereira Lima ${ }^{1}$.

\section{RESUMO}

Objetivo: Estruturar um plano de cuidados de enfermagem por meio da análise das vivências de pessoas no pós-operatório tardio de cirurgia bariátrica. Métodos: Trata-se de uma pesquisa descritiva realizada, com 12 participantes, em duas etapas com abordagens específicas: análise qualitativa do discurso dos participantes; e metodológica para construção do plano de cuidado de enfermagem. Resultados: Os pacientes submetidos à cirurgia bariátrica apresentam como principais dificuldades adaptação ao novo estilo de vida e às mudanças corporais, enquanto que os aspectos mais significativos são a recuperação da autoestima e melhoria da autoimagem corporal. Constatou-se ainda a ausência e o enorme potencial de atuação dos profissionais enfermeiros. Conclusão: Os profissionais enfermeiros devem atuar no acompanhamento dos pacientes submetidos à cirurgia bariátrica por meio de plano de cuidados estruturado, o que possui potencial de otimizar às mudanças do estilo de vida e promover a qualidade de vida, aumentando a taxa de sucesso do procedimento.

Palavras-chave: Cirurgia bariátrica, Enfermagem perioperatória, Discurso, Pesquisa metodológica em enfermagem.

\begin{abstract}
Objective: To structure a nursing care plan by analyzing the experiences of people in the late postoperative period of bariatric surgery. Methods: This is a descriptive research conducted with 12 participants, in two stages with specific approaches: qualitative analysis of the participants' discourse; and methodological for the construction of the nursing care plan. Results: Patients undergoing bariatric surgery have as main difficulties adapting to the new lifestyle and body changes, while the most significant aspects are the recovery of selfesteem and improvement of body self-image. It was also noted the absence and the huge potential for the performance of professional nurses. Conclusion: Professional nurses should monitor patients undergoing bariatric surgery through a structured care plan, which has the potential to optimize lifestyle changes and promote quality of life, increasing the success rate of the procedure.
\end{abstract}

Key words: Bariatric surgery, Perioperative nursing, Address, Nursing methodology research.

${ }_{1}^{1}$ Universidade Federal do Piauí (UFPI), Teresina - PI. *E-mail: ingridmabreu@outlook.com
${ }^{2}$ Universidade Federal do Recôncavo da Bahia (UFRB), Cruz das Almas - BA.

SUBMETIDO EM: 5/2021

ACEITO EM: 5/2021

PUBLICADO EM: 5/2021 


\section{RESUMEN}

Objetivo: Estructurar un plan de cuidados de enfermería mediante el análisis de las vivencias de las personas en el postoperatorio tardío de cirugía bariátrica. Métodos: Se trata de una investigación descriptiva realizada con 12 participantes, en dos etapas con enfoques específicos: análisis cualitativo del discurso de los participantes; y metodológico para la construcción del plan de cuidados de enfermería. Resultados: Los pacientes sometidos a cirugía bariátrica tienen como principales dificultades para adaptarse a los nuevos estilos de vida y cambios corporales, mientras que los aspectos más significativos son la recuperación de la autoestima y la mejora de la autoimagen corporal. También se señaló la ausencia y el enorme potencial para el desempeño de enfermeras profesionales. Conclusión: Las enfermeras profesionales deben monitorear a los pacientes sometidos a cirugía bariátrica a través de un plan de cuidados estructurado, que tiene el potencial de optimizar los cambios en el estilo de vida y promover la calidad de vida, aumentando la tasa de éxito del procedimiento.

Palabras clave: Cirurgia bariátrica, Enfermagem perioperatória, Discurso, Pesquisa metodológica em enfermagem.

\section{INTRODUÇÃO}

Considerada uma epidemia global, a obesidade é uma doença crônica de origem metabólica e/ou genética associada ao excesso de gordura corporal, que pode desencadear doenças psicológicas e fisiológica. A Organização Mundial de Saúde estima que mais de 1,9 bilhões de adultos apresentam excesso de peso e, destes, pelo menos 650 milhões são obesos (WHO, 2017). No Brasil, o número de pessoas obesas também cresceu: em 2006, 42,6\% foram considerados com excesso de peso, em 2016 esse índice subiu para 53,8\% (BRASIL, 2016).

A obesidade apresenta como tratamento invasivo a cirurgia bariátrica, considerada estratégia mais efetiva e duradoura no controle das doenças associadas (CHAIM EA, et al., 2017). Nesse sentido, a indicação formal para esse procedimento reúne idade de 18 a 65 anos, Índice de Massa Corpórea (IMC) maior a $40 \mathrm{~kg} / \mathrm{m}^{2}$ ou $35 \mathrm{~kg} / \mathrm{m}^{2} \mathrm{com}$ uma ou mais comorbidades graves relacionadas com a obesidade e documentação de que os pacientes não conseguiram perder peso ou manter a perda de peso, apesar de cuidados médicos há pelo menos dois anos (ABESO, 2016).

Segundo a Agência Nacional de Saúde Suplementar (ANS, 2017), a cirurgia bariátrica, também denominada de gastroplastia, reúne técnicas destinadas ao tratamento da obesidade e das doenças associadas ao excesso de gordura corporal ou agravadas por ele, sendo capaz de produzir mudanças significativas, tais como redução severa de peso e mudança estética. Por isso, o acompanhamento no pósoperatório é tão importante para a adaptação e para adesão ao tratamento.

Existe a necessidade de orientação dos pacientes no pós-operatório, pois a ausência dessa prática traz complicações comprovadamente negativas, tal como o de reganho de peso (ARAÚJO GB, et al., 2018). De forma complementar, é comum encontrar limitações das equipes de saúde ao acompanhamento dos indivíduos submetidos à cirurgia bariátrica, relacionadas com desconhecimento da complexidade dos cuidados e dificuldade de compreender o caráter subjetivo na adaptação ao novo estilo de vida (BAUER E, et al., 2017).

O cuidado de enfermagem pode representar importante auxílio à adaptação do estilo de vida e manutenção do peso (KORTCHMAR E, et al., 2018). Dessa forma, o profissional enfermeiro tem o potencial de promover educação em saúde com pacientes submetidos ao pós-operatório de cirurgia bariátrica, otimizando as mudanças de estilo de vida e as estratégias de atendimento às necessidades que abrangem 0 ambiente domiciliar e profissional (SOUZA NM e RODRIGUES CA, 2018).

Com a elevada frequência de pessoas com obesidade sendo submetidas à cirurgia bariátrica observa-se a pertinência do conhecimento sobre as potencialidades assistenciais da enfermagem no período do pósoperatório tardio. Contudo, questiona-se: Qual a vivência de pessoas no pós-operatório de cirurgia bariátrica? 
Como o conhecimento dessa vivência pode auxiliar na construção de um plano de cuidados de enfermagem? Objetivou-se, com isso, estruturar um plano de cuidados de enfermagem por meio da análise da vivência de pessoas no pós-operatório tardio de cirurgia bariátrica.

\section{MÉTODOS}

Trata-se de estudo descritivo, executado em duas etapas com abordagens específicas: 1) análise qualitativa do discurso dos participantes; e 2) metodológica para construção do plano de cuidado de enfermagem.

Realizado no interior do estado do Piauí, os participantes foram os indivíduos submetidos à cirurgia bariátrica e se encontravam no pós-operatório tardio, compreendido como o período após sete dias do recebimento da alta com difícil determinação do seu término. Foram incluídos aqueles com idade igual ou maior que 18 anos, que foram submetidos à cirurgia bariátrica em período inferior 30 meses; e excluídos aqueles com diagnóstico de depressão pós-cirúrgica e complicações no pós-operatório autorreferidas.

A amostragem utilizada foi do tipo bola de neve, que se baseia na rede de contatos dos entrevistados para fornecer um conjunto cada vez maior de contatos potenciais (VINUTO J, 2014) e o fechamento da amostra ocorreu quando os dados obtidos passaram a apresentar certa repetição e não houve mais acréscimo de novas informações (FALQUETO J e FARIAS J, 2016), totalizando 12 participantes da pesquisa.

O estudo foi realizado em duas etapas com abordagens específicas. A primeira etapa ocorreu no período de setembro a outubro de 2019, nos períodos diurnos e fins de semanas, no ambiente domiciliar ou em sala reservada na Unidade Básica de Saúde de referência, de acordo com a disponibilidade dos participantes. A execução da amostragem ocorreu pela localização de participantes (sementes) por meio de rede social que, por sua vez, auxiliam o pesquisador com a indicação de novos contatos com as características desejadas e, assim sucessivamente, aumentando a amostragem a cada entrevista (VINUTO J, 2014).

A coleta de informações ocorreu por meio de instrumento semiestruturado, contendo questões sobre dados socioeconômicos e de condições de saúde, principais mudanças efetivas no estilo de vida, dificuldades de adaptação (emocional, autocuidado) e assistência dos profissionais enfermeiros na recuperação após a cirúrgica de bariátrica. Todas as entrevistas tiveram áudios gravados, viabilizando a transcrição das respostas das questões subjetivas. A análise permitiu a identificação de núcleo de sentido, possibilitando a organização das informações, conforme técnica de análise de conteúdo (BARDIN L, 2011).

A segunda etapa ocorreu a partir dos dados empíricos da análise de discurso dos participantes, sendo classificados diagnósticos/resultados e intervenções de enfermagem para pessoas submetidas à cirurgia bariátrica, utilizando os termos constantes na Classificação Internacional para a Prática de Enfermagem (CIPE ${ }^{\circledR}$ ) versão 2019, com base no modelo dos Sete Eixos da CIPE ${ }^{\circledR}$.

O modelo proposto por Calista Roy apresenta fundamento nos comportamentos adaptativos e na relação de adequação em que a vítima pode apresentar, através do estímulo produzido pelo contato com o ambiente estressor. Assim, os comportamentos resultantes serão considerados recursos de acareação aos estímulos que as vítimas possuem, sendo observados a partir de quatro modos adaptativos: fisiológico (respostas físicas dos pacientes aos estímulos ambientais); autoconceito (necessidade de integridade psíquica, enfocando seus valores/expectativas e os aspectos psicoespiritual); desempenho de papel (enfoca os aspectos sociais e seu papel na sociedade); e interdependência (necessidades afetivas, respeito e valores) (ROY C, et al., 2009).

A CIPE ${ }^{\circledR}$, iniciada em 1989 pelo International Council of Nurses (ICN), foi inserida, desde 2008, na Família de Classificações Internacionais da Organização Mundial da Saúde, como Classificação Relacionada. A versão 2019 da CIPE $^{\circledR}$ apresenta 4.475 conceitos, sendo 2.035 conceitos pré-coordenados (relativos a diagnósticos/resultados e intervenções de enfermagem) e 2.440 conceitos primitivos (CLARES JWB, et al., 2020).

Neste estudo utilizou-se as recomendações do ICN e da ISO 18.104:2014 (ISO, 2014) que trata da integração de um modelo de terminologia de referência para a Enfermagem apresentando um termo do Eixo 
Foco e um termo do Eixo Julgamento, podendo incluir termos adicionais. Para a construção das intervenções/ações de enfermagem, devem ser incluídos, obrigatoriamente, um termo do eixo Ação e um termo Alvo, entendido como qualquer um dos conceitos contidos nos demais eixos conforme a necessidade.

Ressalta-se que o estudo seguiu todos os padrões éticos exigidos em pesquisas com seres humanos, tendo sido submetido e aprovado pelo Comitê de Ética e Pesquisa, da Universidade Federal do Piauí, do Campus Amílcar Ferreira Sobral, sob parecer favorável nº 3.415.170/19.

\section{RESULTADOS}

Os participantes possuem idade entre 25 e 57 anos, sendo a maioria com até 40 anos de idade e média de 38,25 anos. Parte representativa é do sexo feminino (11), ensino superior completo (8) e estado civil solteiro (6).

Houve predomínio da gastroplastia utilizando a técnica Sleeve (11), por plano privado de saúde (10), com período de pós-cirúrgico que varia entre sete e 30 meses, com média de 19,5 meses. Considerando o peso anterior à cirurgia, os participantes apresentaram média de $112,5 \mathrm{~kg}$, sendo que após a cirurgia perderam em média 43,5 kg. Logo, a maioria afirma que não teve reganho de peso após a cirurgia (10).

Os participantes relataram, de maneira predominante, que as dificuldades relacionadas à cirurgia bariátrica são a recuperação clínica e mudanças no estilo de vida, conforme relatos:

"Os primeiros dias você tem que tomar de 10 em 10 minutos, depois de meia em meia hora, o copinho de $50 \mathrm{ml}$ de líquido, durante 15 dias. E somente após o décimo sexto dia que passa para alimentação pastosa tem que seguir a regra direitinho, $e$ hoje como normalmente só que bem pouco e só posso beber água após uns trinta minutos porque senão eu vomito" (P9).

"A mudança de hábitos foi a maior dificuldade, e com uma vida de hábitos errados sentir falta de consumir alimentos mais gordurosos porque me sentia mal e até vomitava quando comia" (P1).

Os participantes descreveram como mudanças no estilo de vida mais impactantes na melhoria da qualidade de vida, a recuperação da autoestima e a prática de atividades físicas. A autoestima mostra-se mais evidente no cuidado com o corpo e acesso a roupas. Enquanto que a prática de atividades físicas tornouse uma rotina, conforme relatos:

"Melhora a qualidade de vida, autoestima, passei a me cuidar mais, a me arrumar mais, antes eu não gostava de ir comprar roupas porque sentia vergonha. Minha saúde tá melhor, tenho ânimo para trabalhar, mais mobilidade, não me canso rápido" (P1).

"A principal mudança foi visual quando eu vi que eu consegui entrar em roupa... eu sofri todo tipo de preconceito por ser uma pessoa obesa. Então comecei a me amar, a autoestima subiu; existia uma alegria dentro de mim que eu não sabia, não sei Ihe explicar" (P10).

"Hoje eu vou todos os dias para academia, vou todos os dias pedalar, vou final de semana fazer trilha, e aí houve uma mudança significativa na minha vida" (P5).

As participantes também citaram aspectos relacionados com a feminilidade e autoimagem, tais como a pele flácida e excessiva, a queda de cabelo e as restrições alimentares. Vale ressaltar que a pele flácida é percebida como consequência da cirurgia e com resolução, conforme relatos:

"...Tenho mais muita pele, eu tinha um braço muito largo e hoje eu tenho pele sabe, mas isso é estética que com cirurgia plástica resolve" (P10).

"Até as peles que estão sobrando aqui eu sou conformada com elas, não me incomodam nem diminuem meu bem-estar, me sinto ótima" (P9). 
"Meu cabelo que caiu, caiu muito depois da cirurgia, eu já tinha pouco cabelo e depois caiu agora que tá parando de cair mais um pouco, mas caiu muito, eu me desesperei entendeu 'Vou ficar careca'” (P4).

O apoio de familiares e amigos foi fundamental para tomada de decisão, realização e na adaptação da cirurgia bariátrica. Esse apoio representa um suporte emocional e psicológico para superação dos complexos desafios relacionados à cirurgia, conforme relatos:

"O apoio é fundamental, tive todo o apoio da minha família principalmente do meu pai, da minha mãe e irmã que me acompanharam desde a primeira consulta até a cirurgia e de todos os amigos e demais familiares e isso me ajudou muito durante a adaptação" (P7).

"Eu tive total apoio da minha família graças a Deus... sempre me apoiaram porque sabia que eu tinha esse sonho de diminuir o corpo, o peso para poder viver melhor, para ter uma saúde melhor e para me sentir também melhor" (P4).

O acompanhamento por profissionais de saúde após a cirurgia bariátrica é composto pelo profissional médico gastroenterologista que realizou a cirurgia e a nutricionista. $E$ também pode ocorrer com uma equipe mais ampla que, além desses profissionais, ainda abrangem o profissional psicólogo e médico endocrinologista. Conforme relatos:

"Estou sendo acompanhado pela nutricionista e pelo médico da bariátrica" (P1/P6/P12).

"Os profissionais que me acompanharam foram nutricionistas e psicólogo e o endocrinologista e o próprio gastro" (P8/P9).

Quanto ao acompanhamento por profissional Enfermeiro em ambulatório especializado ou unidade básica, os participantes relataram ausência da atuação desse profissional. No entanto, houve um relato equivocado que o profissional médico contemplaria todo o acompanhamento necessário e outro que lamenta ausência de projeto envolvendo o profissional Enfermeiro, conforme relatos:

"Não procurei porque assim eu acho que o médico bariátrico já abrange tudo, entendeu. Por exemplo, passa exame caso você esteja fraco de vitamina de alguma coisa então é tudo com ele; por isso que eu não procurei outros profissionais" (P4).

"O estado infelizmente não tem nenhum projeto social para que enfermeiros para que a UBS Abrace a galera da cirurgia bariátrica" (P10).

A identificação das necessidades de saúde das pessoas submetidas à cirurgia bariátrica subsidia 0 desenvolvimento de plano de cuidados. O plano proposto neste estudo baseou-se na Teoria Adaptativa de Roy, devido à necessidade de respostas adaptativas, e norteará a atuação de profissionais Enfermeiros. 
Quadro 1 - Plano de cuidados para pessoa submetida à cirurgia bariátrica, fundamentada na Teoria Adaptativa de Roy.

\begin{tabular}{|c|c|}
\hline Diagnósticos/resultados de enfermagem & Intervenções de enfermagem \\
\hline \multicolumn{2}{|c|}{ Modo Adaptativo Fisiológico } \\
\hline Condição Nutricional, Prejudicada (10012804) & Gerenciar Condição Nutricional (10036013) \\
\hline $\begin{array}{l}\text { Risco de Condição Nutricional, Prejudicada } \\
\text { (10037224) }\end{array}$ & $\begin{array}{l}\text { Promover Condição Nutricional, Positiva } \\
(10050920)\end{array}$ \\
\hline Condição Nutricional, Melhorada (10035569) & $\begin{array}{l}\text { Auxiliar na Ingestão de Alimentos ou Líquidos } \\
\text { (10037269) }\end{array}$ \\
\hline Desequilíbrio de Líquidos (10042335) & $\begin{array}{l}\text { Avaliar Resposta à Terapia com Líquidos (ou } \\
\text { Hidratação) (10007176) }\end{array}$ \\
\hline $\begin{array}{l}\text { Falta de Conhecimento sobre Regime de Líquidos } \\
(10042123)\end{array}$ & $\begin{array}{l}\text { Colaborar na Terapia com Líquidos (ou Hidratação) } \\
\text { (10030948) }\end{array}$ \\
\hline Ingestão de Líquidos, Prejudicada (10029873) & $\begin{array}{l}\text { Gerenciar Terapia com Líquidos (ou Hidratação) } \\
\text { (10042096) }\end{array}$ \\
\hline $\begin{array}{l}\text { Comportamento de Ingestão de Alimentos, } \\
\text { Compulsivo (10004896) }\end{array}$ & $\begin{array}{l}\text { Medir (ou Verificar) Ingestão de Líquidos } \\
\text { (10039245) }\end{array}$ \\
\hline Ingestão de Alimentos, Excessiva (10000682) & Monitorar Ingestão de Líquidos (10035303) \\
\hline $\begin{array}{l}\text { Ingestão de Alimentos, Insuficiente (ou Deficitária) } \\
\text { (10000607) }\end{array}$ & Oferecer Bebida (ou Líquidos) (10050313) \\
\hline $\begin{array}{l}\text { Risco de Ingestão de Alimentos, Insuficiente (ou } \\
\text { Deficitária) (10023021) }\end{array}$ & Orientar sobre Ingestão de Líquidos (10032939) \\
\hline Vômito (10025981) & Monitorar Ingestão de Alimentos (10036614) \\
\hline $\begin{array}{l}\text { Função do Sistema Gastrointestinal, Prejudicada } \\
\text { (10022931) }\end{array}$ & $\begin{array}{l}\text { Orientar sobre Padrão de Ingestão de Alimentos } \\
(10032918)\end{array}$ \\
\hline $\begin{array}{l}\text { Risco de Função do Sistema Gastrointestinal, } \\
\text { Prejudicada (10046431) }\end{array}$ & Avaliar Condição Gastrointestinal (10034007) \\
\hline \multicolumn{2}{|c|}{ Modo Adaptativo Autoconceito } \\
\hline Autoimagem, Negativa (10022724) & Apoiar Imagem Corporal, Positiva (10044 \\
\hline Baixa Autoestima (10029507) & Orientar sobre Autocuidado (10045014) \\
\hline Déficit de Autocuidado (10023410) & Promover Autocuidado (10026347) \\
\hline- & Auxiliar no Autocuidado (10035763) \\
\hline \multicolumn{2}{|c|}{ Modo Adaptativo Desempenho de Papel } \\
\hline $\begin{array}{l}\text { Aceitação da Condição de Saúde, Prejudicada } \\
\text { (10029480) }\end{array}$ & $\begin{array}{l}\text { Promover Aceitação de Condição de Saúde } \\
\text { (10037783) }\end{array}$ \\
\hline $\begin{array}{l}\text { Falta de Conhecimento sobre Regime Dietético } \\
(10021939)\end{array}$ & Promover Adesão ao Regime Dietético (10032449) \\
\hline Não Adesão ao Regime Dietético (10022117) & $\begin{array}{l}\text { Orientar Família sobre Regime Dietético } \\
(10026525)\end{array}$ \\
\hline Tolerância à Dieta (10036370) & Reforçar Regime Comportamental (10039002) \\
\hline- & Facilitar Adesão ao Regime (10036273) \\
\hline- & Orientar sobre Dieta (10046533) \\
\hline \multicolumn{2}{|c|}{ Modo Adaptativo Interdependência } \\
\hline Adaptação, Prejudicada (10022027) & Orientar Técnicas de Adaptação (10023717) \\
\hline $\begin{array}{l}\text { Risco de Condição Psicossocial, Prejudicada } \\
(10038424)\end{array}$ & $\begin{array}{l}\text { Promover Condição Psicológica, Positiva } \\
\text { (10032505) }\end{array}$ \\
\hline Condição Psicológica, Prejudicada (10038411) & Identificar Condição Psicossocial (10044241) \\
\hline
\end{tabular}

Fonte: Rocha RMF, et al., 2021. 


\section{DISCUSSÃO}

Considerando os participantes do estudo, houve predomínio do sexo feminino, com média de 39 anos de idade, corroborando com outro estudo realizado com pacientes bariátricos (MAIA RP, et al, 2018). Neste sentido, as mulheres são quatro vezes mais suscetíveis a procurar a cirurgia bariátrica do que os homens, embora a distribuição da obesidade independa do sexo, o que está associada com maior preocupação com aparência física e estética (CARVALHO AS e ROSA RS, 2019).

Os obesos frequentemente relatam menor qualidade de vida em aspectos como autoestima, relacionamentos amorosos, vida no trabalho e socialização, o que pode ser compreendido como fatores estimuladores para realização da cirurgia bariátrica entre pessoas jovens, solteiras e com elevada escolaridade, à medida que a obesidade gera problemas nas relações sociais (BAUER E, et al., 2017).

A técnica cirúrgica mais utilizada foi Sleeve, o que diverge da técnica mais comum (Bypass) na rede pública de saúde (CARVALHO AS e ROSA RS, 2019). Trata-se de procedimento restritivo e metabólico, que provoca uma boa perda de peso, além de ter boa eficácia sobre o controle da hipertensão e de doenças dos lipídeos (ANS, 2017). Apesar da escolha da técnica cirúrgica ser compartilhada entre o médico e o paciente, sabe-se que a técnica Sleeve é muito comum entre os pacientes, devido à rápida perda de peso e ampla indicação em rede social.

O uso predominante do plano privado de saúde para realização da cirurgia bariátrica pode estar relacionado com baixa oferta na rede pública de saúde, no qual o período de espera pode variar entre 1 e 4 anos. Enquanto que o peso médio anterior à cirurgia coincide, mas a perda média de peso foi inferior ao achado em outro estudo, fato relacionado ao período pós-cirúrgico (CARVALHO AS e ROSA RS, 2019).

Segundo a Agência Nacional de Saúde Suplementar a realização correta da intervenção cirúrgica não impede futuro ganho de peso se o paciente não prosseguir com o acompanhamento e mantiver disciplina no tratamento (ANS, 2017). Entretanto, os participantes apresentaram perda média de peso significativa, em conformidade com estudo no qual a cirurgia bariátrica tem propiciado alta perda de peso, além de resolução de comorbidades e melhora na qualidade de vida (MAIA RP, et al., 2018).

A adaptação aos novos hábitos alimentares constitui um desafio recorrente aos pacientes submetidos à cirurgia bariátrica. Por isso, a reeducação alimentar deve iniciar ainda no pré-operatório, pois o comportamento alimentar inadequado é fator determinante para o insucesso da intervenção cirúrgica, já que sem uma mudança radical no comportamento alimentar, a cirurgia perde o potencial emagrecedor (JESUS $A D$, et al., 2017).

Quanto aos aspectos emocionais, os achados coincidem com o estudo Maia RP, et al. (2018) em que houve melhora significativa da qualidade de vida, bem como do autoconceito, da autoestima, dos sentimentos, da satisfação, da vida laboral e da capacidade de realizar atividades físicas, sociais e sexuais. De maneira geral, a cirurgia bariátrica produz um impacto positivo no comportamento psicossocial.

A descrição da satisfação no acesso às roupas revela melhoria da autopercepção da imagem corporal. Quanto mais precoce for o quadro de obesidade, observa-se uma maior incidência de preocupação com a imagem corporal, especialmente entre as mulheres, o que revela um risco de adoecimento psíquico, devido à cobrança social (CARVALHO AS e ROSA RS, 2019).

Em pesquisa realizada para comparar antes e após a cirurgia bariátrica, observou-se que houve aumento da quantidade de pessoas que aderem à prática regular de atividades físicas, compreendida como hábito componente do estilo saudável, o que coincide com os achados deste estudo (MAIA RP, et al., 2018).As mudanças corporais citadas mostram que os pacientes podem ter uma perda de peso adequada e recuperação da autoestima, mas podem se apresentar insatisfeitos com o impacto da operação nos mais diversos fatores que envolvem a qualidade de vida, na quantidade da ingesta alimentar diária, e na repercussão do pós-operatório na imagem corporal (CARVALHO AS e ROSA RS, 2019).

A imagem corporal após a cirurgia bariátrica, com significativa perda de peso, inclui o excesso de pele flácida e comumente ocorre também a perda de cabelo, como complicação clínica, o que afeta a feminilidade 
e agrega o risco de depressão, devido à dificuldade em aceitar a nova imagem corporal e adaptação póscirúrgica (CASTANHA CR, et al., 2018). Evidenciando a necessidade de acompanhamento profissional nas mudanças corporais e de estilo de vida.

Os resultados mostram que a participação e o apoio familiar são essenciais na decisão pela cirurgia e póscirúrgico, pois as mudanças no estilo de vida e hábitos alimentares precisam ser contínuas e rigorosas. Durante todo o processo, entre a indicação da cirurgia e perda de peso no pós-cirúrgico, o paciente precisa estar cercado de pessoas que o compreendam e amparem (BAUER E, et al., 2017).

Os achados do estudo apontam um acompanhamento focado no profissional médico, entretanto o tratamento da obesidade é complexo e exige uma abordagem multidisciplinar, com o objetivo de interagir os saberes e qualificar a assistência (ARAUJO GB, et al., 2018). Dessa forma, as equipes multiprofissionais de saúde precisam investir nos encontros com o paciente após a cirurgia bariátrica, compartilhando decisões e promovendo sua autonomia com vistas à manutenção do peso (KORTCHMAR E, et al., 2018).

Torna-se fundamental que outros profissionais, como os enfermeiros atuem nos cuidados pós-operatórios, por meio de protocolo de cuidados, orientando sobre as mudanças no estilo de vida e contribuindo para bemestar do paciente e sucesso da cirurgia. Esses profissionais devem ofertar cuidados envolvidos na educação em saúde, visando o auxílio ao paciente na sua adaptação a nova rotina. Entretanto, outro estudo aponta que os mesmos não possuem conhecimento sobre os protocolos operacionais padrão e sobre 0 atendimento ao paciente bariátrico (SANTOS TR, et al., 2018).

Observa-se uma assistência de enfermagem bem estruturada no pré-operatório e no pós-operatório imediato, desenvolvendo um protagonismo na recuperação com menor risco de complicações de pacientes submetidos à cirurgia bariátrica e favorece a identificação das necessidades do paciente (PEZZIM IM, et al., 2020). Entretanto, constata-se ausência de protocolo específico para o paciente no pós-operatório de cirurgia bariátrica (DOLEN F, et al., 2020).

Para que o profissional enfermeiro exerça seu potencial no acompanhamento do pós-operatório do paciente submetido à cirurgia bariátrica, precisa ser capacitado quanto ao acompanhamento ambulatorial e na atenção primária à saúde, oferecendo uma assistência que contribua para o efetivo sucesso da cirurgia, mudança de comportamento e melhoria da qualidade de vida (DOLEN F, et al., 2020).

O sucesso da cirurgia bariátrica está relacionado com a redução e manutenção do peso corporal que, por sua vez, está condicionada à capacidade de adaptação do paciente às mudanças no comportamento e estilo de vida. $O$ estudo apresenta como limitações um grupo de pacientes submetidos à cirurgia bariátrica num período inferior há 36 meses, tempo mínimo indicado para reganho; uso exclusivo da Teoria Adaptativa de Roy, como referencial teórico de enfermagem; e ausência de validação do protocolo de cuidados de enfermagem. O plano de cuidados de enfermagem representa uma importante estratégia de atuação desses profissionais no acompanhamento do pós-operatório de pacientes submetidos à cirurgia bariátrica que deve ser incorporada na prática clínica. Considera-se, ainda, pertinente a continuidade do estudo com a realização do acompanhamento de pacientes submetidos a esse procedimento por enfermeiros utilizando o protocolo de cuidados com enfoque na promoção da saúde.

\section{CONCLUSÃO}

Os pacientes submetidos à cirurgia bariátrica apresentam como principais dificuldades a adaptação ao novo estilo de vida, adoção hábitos alimentares saudáveis e adequação às mudanças corporais, enquanto que os aspectos mais significativos são a melhoria da autoimagem corporal e da feminilidade, bem como recuperação da autoestima e desenvolvimento de atividades físicas. Constatou-se a ausência, em contraste com o grande potencial de atuação dos profissionais enfermeiros no reconhecimento das necessidades de saúde desses pacientes e, respectivo, estabelecimento de estratégias de adaptação. Portanto, os profissionais enfermeiros devem atuar no acompanhamento dos pacientes submetidos à cirurgia bariátrica por meio de plano de cuidados estruturado, auxiliando nas adaptações às mudanças do estilo de vida e promoção da qualidade de vida. 


\section{REFERÊNCIAS}

1. AGÊNCIA NACIONAL DE SAÚDE SUPLEMENTAR (ANS). Manual de diretrizes para o enfretamento da obesidade na saúde suplementar brasileira. 2017. Disponível em: https://www.ans.gov.br/images/final_obesidade_26_12.pdf. Acessado em: 10 de abril de 2021.

2. ARAÚJO GB, et al. Clinical and epidemiological profile of patients undergoing on bariatric surgery. Para Res Med J, 2018; 1(4):e38.

3. Associação Brasileira para o Estudo da Obesidade e da Síndrome Metabólica Diretrizes brasileiras de obesidadeABESO - Associação Brasileira para o Estudo da Obesidade e da Síndrome Metabólica. 2016]. Disponível em: https://abeso.org.br/wp-content/uploads/2019/12/Diretrizes-Download-Diretrizes-Brasileiras-de-Obesidade-2016.pdf. Acessado em: 22 de fevereiro de 2021.

4. BARDIN L. Análise de conteúdo. São Paulo: Edições 70, 2011

5. BAUER E, et al. The complexity of obesity before and after bariatric surgery. RBONE - Revista Brasileira De Obesidade, Nutrição E Emagrecimento, 2017; 11(68): 653-660.

6. CARVALHO AS, ROSA RS. Bariatric surgeries performed by the Brazilian National Health System in the period $2010-$ 2016: a descriptive study of hospitalizations. Epidemiol. Serv. Saúde, 2019; 28 (1): e2018260.

7. CASTANHA CR, et al. Evaluation of quality of life, weight loss and comorbidities of patients undergoing bariatric surgery. Rev. Col. Bras. Cir, 2018; 45(3): e1864.

8. CHAIM EA, et al. Preoperative multidisciplinary program for bariatric surgery: a proposal for the Brazilian Public Health System. Arq Gastroenterol, 2017; 54(1):70-4.

9. CLARES JWB, et al. International Classification for Nursing Practice in Brazilian dissertations and theses. Rev. Eletr. Enferm, 2020; 22:56262.

10. DOLEN F, et al. Nursing performance in observative patient care in the positive surgery of bariatric surgery. Revista Brasileira de Obesidade, Nutrição e Emagrecimento, 2020; 14(87):550-9.

11. FALQUETO J, FARIAS J. Saturação teórica em pesquisas qualitativas: relato de uma experiência de aplicação em estudo na área de administração. CIAIQ, 2016; 3(1):560-9.

12. JESUS AD, et al. Food behavior of pre and post-bariatric surgery patients. Revista brasileira de obesidade, nutrição e emagrecimento, 2017; 11(63): 187-96.

13. KORTCHMAR E, et al. Recuperación del peso después de la cirugía bariátrica: un enfoque de la fenomenología social. Actapaul. Enferm, 2018; 31(4):417- 22.

14. MAIA RP, et al. Nutritional profile and quality of life assessment after bariatric surgery in a public hospital of Goiania, Brazil. Demetra, 2018; 13(1); 147-64.

15. Ministério da Saúde- Brasil. Vigitel Brasil 2016 Saúde Suplementar: vigilância de fatores de risco e proteção para doenças crônicas por inquérito telefônico. Brasília (DF): Ministério da Saúde. 2017. Disponível em: https://www.ans.gov.br/images/Vigitel_Saude_Suplementar.pdf3. Acessado em: 25 de agosto de 2019.

16. PEZZIM IM, et al. Anxiety contributes to increasing the degree of dependence on nursing care in the immediate postoperative of bariatric surgery. Rev Min Enferm, 2020; 24:e-1321.

17. ROY C, et al. O Modelo de Adaptação de Roy e Pesquisa: Perspectiva Global. Sage Journals, 2009; 22(3):209-11.

18. SANTOS TR, et al. Conhecimento da equipe de enfermagem relacionado à cirurgia Bariátrica em um hospital de ensino. Revista Saúde e Comunidade UNESPAR, 2018; 1(1): 12-9.

19. SOUZA NM, RODRIGUES CA. Cuidados de Enfermagem no Pós-operatório de Gastroplastia: Uma Revisão Bibliográfica. 2018. Disponível em: http://repositorio.unis.edu.br/bitstream/prefix/325/1/nathalia.pdf. Acessado em: 22 de fevereiro de 2021.

20. VINUTO J. Snowball sampling in qualitative research an open debate. Temáticas, 2014; 22(44): 203-20.

21. WORLD HEALTH ORGANIZATION (WHO). Controlling the global obesity epidemic. 2017. Disponível em: https://www.who.int/activities/controlling-the-global-obesity-epidemic. Acessado em: 22 de fevereiro de 2021. 